\title{
Research on Evaluation of Weaponry Equipment Quality in Using Stage
}

\author{
Bo Wang \\ School of Ordnance Engineering College, Shijiazhuang 050000, China \\ atcwangbo@sohu.com
}

\begin{abstract}
The quality of weaponry equipment is reflected in using stage. Evaluate the quality of weaponry equipment in using stage provides a reference for how to do the work of application and management, helps higher administrative departments to master the working condition, and helps manufacturers to optimize and improve the equipment performances. This paper mainly introduce the concept of weaponry equipment quality in using stage, and the methods and theories of quality evaluation. Through this paper, provide a theoretical basis for building up an evaluation index system for weaponry equipment quality in using stage.
\end{abstract}

Keywords: weaponry equipment, use stage, evaluation of quality.

\section{The concept of weaponry equipment quality in using stage}

\subsection{Quality of weaponry equipment}

The concept of quality is the degree to which a set of inherent characteristics fulfils requirements. And the quality of weaponry equipment, is means a set of inherent characteristics of weaponry equipment that could meet the requirements of battle, training and support action for army, and whether it could satisfied the performance criteria of itself.

The quality of the commodity refers to the sum of the various properties required to satisfy its function, under a certain conditions of use. Weaponry equipment is a special commodity, it is used for army, and its purpose is to complete the army's battle, training and support tasks. Therefore, evaluate the quality of weaponry equipment, we should base on the degree of customers' satisfaction, in order to determine whether the weaponry equipment can complete the army's battle, training and support tasks, and whether it can satisfy the users for its using requirement.

\subsection{Quality of weaponry equipment in using stage}

The process of weaponry equipment from the allotment of troops to scrap, is called using stage. And during this stage, it is used in various practical activities, for example: battle, training, support and other tasks. Using stage is the stage of reflect its quality and other value of itself, is the stage to judge the weaponry equipment have a high quality or not, and is the stage to work and meet the needs of users. Quality of weaponry in using stage, directly affect the combat effectiveness of the army. Often, most of the faults of the equipment is occurs in using stage, and through these problems can reflect the user satisfaction and demand with the equipment.

Normally, we are mainly understand the concept of weaponry equipment quality from two aspects: confirmative quality and applicable quality. The confirmative quality is measure and evaluate the degree of compliance, based on the technical index, the tactical index and the performance standard, if the equipment have a high degree of compliance with various standards, we call it have a high confirmative quality, on the contrary, the confirmative quality is low. The applicable quality is measure and evaluate the satisfaction of the user, based on the usage requirements of the equipment and the degree that the equipment meet the needs of users, if the equipment meet the needs of users with a high degree, we call it have a high applicable quality, on the contrary, the applicable is low.

In conclusion, this paper holds that in order to evaluate the weaponry equipment quality and build a evaluating index system of weaponry equipment quality, we should follow two principles. On the one hand, is the principle of compliance, equipment should suit various standards that developed during its design and production stage. On the other hand, is the principle of applicability, the degree that equipment meet the needs of users in using stage. 


\section{Research and Analysis on the quality of equipment in using stage}

In the modern battlefield, high-tech weaponry equipment are widely used, and its quality has an important influence to the war, thus, the requirement for the quality of weaponry equipment is getting higher and higher. There are two contents in the working of weaponry equipment evaluation, one is have a scientific, objective and accurate evaluation, in order to grasp the quality status of weaponry equipment, find out the faults and hidden dangers timely, and take appropriate measures to maintenance and improvement in time, ensure the weaponry equipment keep in a good condition. On the other hand, through the quality evaluation of weaponry equipment, different types of weaponry equipment can be compared, and then provide a reference for the higher leading department to select reliable equipment for army, make a feasible training plan and improve the equipment performance.

\subsection{The principle of quality evaluation of equipment in using stage}

"Quality" is defined as the degree to which a set of inherent characteristics fulfils requirements. It is can be seen from the definition of quality, the basic of it is "satisfy the requirement", as mentioned here, requirement is not only the needed of users, but also the requirement that proposed during design and production stage. The meaning of "inherent characteristics" is a designed index that according to the product's characteristics, use and needed of users, used to evaluate, verify and manage the product quality. And the evaluation criteria are determined according to the degree of user's satisfaction.

\subsection{The basis of equipment quality evaluation in using stage}

Evaluation of the quality of weaponry equipment in using stage, is from the perspective of the user, and including the parameters for evaluation in combat capability, supportability, maintainability, operability, etc. The evaluation results can be used as a fast, effective and accurate feedback information, provide a support for the higher department to supervise the status of weaponry equipment in life cycle, and improve the level of weaponry equipment construction. Thus, improve the quality of equipment continuously, and give full play to the effectiveness of equipment.

The quality of weaponry equipment directly affects its training, using and management. A certain type of equipment whether is comfortable for ride, convenient for operate and maintenance, adapt to different environments and other aspects, are all affected by its quality. Do the working of equipment quality evaluation, is to judge and make decisions on these parameters and problem.

Therefore, we should do the working of equipment quality evaluation based on the technical standard of the equipment and users' satisfaction in use.

\subsubsection{Technical standard}

Philip B. Crosby, a world famous expert in quality management, defined quality as "The quality of a product or service refers to the characteristics of which they can be measured or determined that meeting specification standard". This definition is defined from the point of view of producer, pointed out the standard that the equipment should meet in the process of production and use, we call it the technical standard of the product. This is the basic standard to evaluate the quality of equipment, help the user to manage the quality of equipment, and also as an evaluation basis for evaluate the inherent quality characteristics of equipment.

\subsubsection{User satisfaction}

In the above, we refer to the concept of confirmative quality and applicable quality, this two concept is to describe the quality from two different point of view. In order to synthesize these two concepts, evaluate the quality of equipment from all aspects, we use the concept of user satisfaction, and use it for evaluation. In fact, measure the quality of weaponry equipment is based on the quality characteristics of the equipment whether can meet the needs of the army and the degree of user satisfaction. Therefore, the starting point and end result of weaponry equipment evaluation is to meet the needs of users, and reflected through the user satisfaction with the use of equipment.

W. Edwards. Deming, a world famous expert in quality management, explain the concept of quality in the book of recovered from the crisis: quality must be defined by customer satisfaction, it is multidimensional, and it is determined by the combination of customer satisfaction and product characteristics. Joseph M. Juran, an expert in quality management, defined the quality as "fitness for use", it is means that the products should meet customer's needs throughout the period of use. 
Ishikawa kaoru, a Japanese quality management expert, his opinion of quality is "quality equals customer satisfaction, and must be comprehensively defined". Thus, we know that quality is not single but multidimensional, it is a combination of customer satisfaction and product characteristics.

User satisfaction is the degree to which the user is satisfied with the use of the product, it is the subjective perception of the use of equipment, and it is the degree that equipment satisfied user's requirement. In the process of equipment quality evaluation, we should collect information of satisfaction of equipment use that provided by each position operator, in order to let the results obtained are more comprehensive, systematic and true, and provide accurate and detailed data for equipment quality evaluation.

\subsection{The purpose of weaponry equipment usage quality evaluation}

The problem of the quality of weaponry equipment in using stage refers to the phenomenon that the equipment performance in using stage is not in conformity with the actual needs and is not suitable. Through the evaluation of the equipment quality in using stage, not only to find the problem that occurred in the course of use, and also to find the quality problem caused by the problem that existing in the design and production process.

\section{Evaluation index system and evaluation methods}

\subsection{Principles of evaluation}

\subsubsection{Principle of comprehensiveness}

The equipment quality evaluation involves many kinds of equipment, large quantity and complex structure, different equipments have a different uses, different structure of the equipment has a different tasks, and different user have different usage requirements of equipment. Thus, the quality evaluation index system should be comprehensive in order to adapt to different usage environment and usage requirements, make a reasonable and comprehensive evaluation, and ensure that the evaluation results are true and reliable.

\subsubsection{Principle of hierarchy}

Different weaponry equipment of formation, structure and scale, have different functions, roles and intentions. And different users have different requirements for the use of weaponry equipment. With the position of equipment users from low to high, the number of equipment from less to more, the type of equipment from single to complex, the quality of equipment evaluation also from micro to macro, from local to comprehensive changes. Therefore, it is necessary for us to consider the vertical and horizontal consideration in the process of building the equipment quality evaluation index system, in order to meet the different needs of different users.

\subsubsection{Principle of openness}

With the development of modern science and technology, the renewal speed of weaponry equipment is accelerated, and the demand for the use of weaponry equipment is also increasing. With the continuous delivery of new equipment, the original equipment system is constantly improved and upgraded, so that weaponry equipment is developed and improved unceasingly, the function of the equipment is more and more, the combat capability is more and more strong, and the system of equipment is more and more complex. Therefore, in the process of building equipment quality evaluation index system, we should take into account the principle of openness, make sure the system may scalable, in order to let the evaluation system can be improved with the renewal and progress of the weaponry equipment.

\subsection{Subject of evaluation}

The subject of evaluation is human, is the direct user of weaponry equipment in using stage, mainly including operators, maintenance personnel, management personnel, etc. We can get the satisfaction of different people on the operation, use, maintenance and management of the equipment by the way of questionnaire or direct dialogue, and then summarize, merge, classify and analyze the obtained information, used to provide reference for evaluation. 


\subsection{Object of evaluation}

Object of evaluation is weaponry equipment, it can be a single equipment, and also can be an organizational system, a series of equipment. Analysis according to logical relationship, structure and scale of weaponry equipment, we can divide it into three levels: micro, meso and macro, the macro level is the integration of micro and meso, it is possible to get the results of the overall evaluation by the evaluation of different individual equipment or system. The relationship among them is: the macro level is the integration of micro and meso, it can reflect the overall quality level of a whole or a series of equipment; Micro and meso is the basis of macro, it is the concrete and detailed evaluation of macro evaluation.

For example, the equipment of a certain army can be divided into small, medium and large levels according to its combat tasks, construction and scale. We can achieve the purpose of the comprehensive evaluation of equipment from the individual equipment to the weaponry equipment system, from specific to comprehensive, from micro to macro, through the evaluation of these three levels. Through a complete and comprehensive evaluation system of weaponry equipment quality, the quality of different levels can be evaluated, which can objectively reflect the needs and satisfaction of equipment quality of different levels. The evaluation results can provide reference for different level department to develop equipment training plan and management plan, and also provide reference for equipment producing department to improve equipment performance and applicability.

\subsection{The main evaluation methods}

It is important to choose a scientific evaluation method throughout the evaluation process. Choosing a reasonable and scientific evaluation method can help to get a correct and valuable evaluation results. By reading a large number of literature, concluded that the commonly used evaluation methods can be divided into three categories: qualitative evaluation method, quantitative evaluation method and synthesis evaluation method.

\subsubsection{Qualitative evaluation method}

Qualitative evaluation method is a kind of evaluation method of evaluating by experience and the ability of observation, analysis and logical judgment. This method is usually based on the existing information, evaluator score or make intuitive judgments on the evaluation object, and then put forward the views of the summary, obtained the final evaluation result.

Advantages: Without the limitation of the statistical data, and it could give full play to the wisdom and experience of the evaluator, so as to avoid the one-sidedness and boundedness that resulting from inadequate or inaccurate statistical data.

Disadvantages: The evaluation process is affected by many random factors, and the evaluation results are easily influenced by the subjective consciousness, experience and knowledge of the evaluator, easy to have individual subjective tendency and on sidedness.

\subsubsection{Quantitative evaluation method}

The quantitative evaluation method is base on the statistical data obtained from various tests, construct a mathematical model according to the evaluation index system, and obtain the result by mathematical method or computer. The evaluation result of this method is usually expressed in number.

Advantages: The evaluation process is based on the data, and the evaluation result is objective. Using a scientific computational methods to evaluate, to avoid the influence of individual subjective tendency and one-sidedness. The evaluation result is scientific and reliable.

Disadvantage: In the case of the evaluation object is more complex, some evaluation content is difficult to express in quantity.

\subsubsection{Synthesis evaluation method}

Synthesis evaluation method is the combination of qualitative evaluation method and quantitative evaluation method, absorb the advantages of both, but also to make up for the shortcomings of both. 


\section{Summary}

With the continuous development of science and technology, the battlefield environment is changing rapidly, the quality requirement of the weaponry equipment is also getting higher and higher. The quality of weaponry equipment is reflected in using stage, and have important influence on the operation, training and management of military equipment. Therefore, it is particularly important to complete the quality evaluation of weaponry equipment in using stage, feedback the quality of weaponry equipment objective and true.

\section{References}

[1] Li Weizhong. To Study on the Comprehensive Evaluation System and Method of Commodity Quality [J]. Value Engineering, 2006, (9).

[2] Li Zhao, Wang Lizhi, Tian Wu. Macro Evaluation Index System of Product Quality [J]. Economic Herald, 2010, (11).

[3] Zhu Kejia, Jiang Jiadong. Research of National Quality Evaluation Index System [J]. Aeronautic Standardization \& Quality, 2012, (6).

[4] Yang Ying, Tang Wanjin, Gao Xiaohong. Establishment of Index System of Macro-Quality Estimation [J]. Standard Science, 2009, (3).

[5] Zhang Yifei. Analysis on the Construction of Commodity Quality Evaluation Index System [J]. Market Modernization, 2013, (29).

[6] Liang Xinhua, Wang Duanmin. Overview of Evaluation of Equipment Maintenance Quality [J]. China Plant Engineering, 2006, (4). 